\title{
Malignant pleural mesothelioma: current perspectives
}

\begin{abstract}
The objectives of this study are to review epidemiology, novel methods of detection, novel diagnostics, and novel therapeutic options of malignant pleural mesothelioma (MPM) in the literature that were published between 1977 and 2019. Malignant pleural mesothelioma, associated with prolonged respirable-asbestos-fiber exposure is a rare cancer with constantly increasing incidence and poor prognosis due to lacking the effective treatment options. The median survival ranges from 8 to 14 months. Sarcomatoid histological subtype has the worst prognosis. Video-assisted thoracoscopy plus mediastinoscopy is the current gold standard for staging malignant pleural mesothelioma and is superior to computerized tomography of the chest for assessing the tumor size and suspected nodal metastases. Several circulating biomarkers are detected in MPM patients, such as mesothelin, osteopontin, fibulin-3, high mobility group B1, vascular endothelial growth factor, reactive oxygen species, reactive nitrogen species, micro-ribonucleic acids, tumor deoxyribonucleic acid, etc.Surgery is controversial and restricted to patients with early stage disease and good functional status. There is still no curative treatment whereas chemotherapy is the only treatment modality that improve patients' survival. Radiotherapy is beneficial as a palliative treatment and an adjuvant to chemotherapy and surgery in the context of trimodality treatment. In conclusion, there is potential for the development of biomarkers and radiological imaging in the years to come. Several novel therapeutic options are under studied and it is likely to involve highly personalized treatment. Its incidenceis expected to decrease in the next decade.
\end{abstract}

Volume 7 Issue 2 - 2020

\author{
Attapon Cheepsattayakorn, ${ }^{1,2}$ \\ Ruangrong Cheepsattayakorn, ${ }^{3}$ Supawan \\ Manosoontorn, ${ }^{2}$ Vijaya Bhakskara Reddy \\ Mutha ${ }^{2}$ \\ 'IOth Zonal Tuberculosis and Chest Disease Center, Thailand \\ ${ }^{2}$ Faculty of Public Health, St Theresa International College, \\ Thailand \\ ${ }^{3}$ Department of Pathology, Faculty of Medicine, Chiang Mai \\ University, Thailand
}

\section{Correspondence: Attapon Cheepsattayakorn, I0th Zonal Tuberculosis and Chest Disease Center, 143 Sridornchai Road Changklan Muang Chiang Mai 50100,Thailand, Tel +66 53 |40767/66 53 276364, Fax +66 53 |40773/66 53 273590, Email Attapon1958@gmail.com}

Received: January 01, 2020 | Published: April 02, 2020

Keywords: pleural, mesothelioma, malignant, diagnosis, treatment, epidemiology

Abbreviations: BALF, bronchoalveolar lavage; BAP1, BRCA-associated protein 1; CFAP45, Cilia and flagella associated protein 45; CT, computed tomography; CTCs, circulating tumor cells; ctDNA: circulating tumor deoxyribonucleic acid; DNA, deoxyribonucleic acid; FDG, 18-fluoro-deoxy-glucose; HMGB1, high mobility group B1 ; miRNAs, micro-Ribonucleic acids; MPM, malignant pleural mesothelioma; MRI, magnetic resonance imaging ; MSLN, mesothelin; PDGF, platelet-derived growth factor; PET-CT, positron-emission technology-computed tomography;RNS, reactive nitrogen species; ROS, reactive oxygen species; RR2, ryanodine receptor 2; SETDB1: set domain bifurcated 1; SETD2, set domain containing 2; SMRP, soluble mesothelin-related peptide; TGF- $\beta$, tumor growth factor- $\beta$; TNM, tumor-nodes-metastases;UICC, the union for international cancer control; UK, united kingdom; ULK2, Unc-like autophagy activating kinase; USA, united states of america; US FDA, united states food and drug administration; VATS, videoassisted thoracoscopic surgery; VEGF, vascular endothelial growth factor

\section{Objectives of the study}

The objectives of this study are to review epidemiology, novel methods of detection, novel diagnostics, and novel therapeutic options of malignant pleural mesothelioma (MPM) in the literature that were published between 1977 and 2019.

\section{Introduction}

Malignant pleural mesothelioma, a tumor originated from the submesothelial or mesothelial cells of pleura, pericardium, or peritoneum accounts for more than $80 \%$ arising from the pleura that the majority are male patients. ${ }^{1,2}$ MPM, a rare cancer with increasing incidence and poor prognosis because of lacking the effective therapeutic interventions. ${ }^{1,3,4}$ MPM is associated with previous longterm asbestos exposure of about 40 years of latency period. ${ }^{5-9}$ The total incidence is highest in the UK and USA whereas the global incidence has increased constantly over the past decade, and is predicted to reach the estimated peak in $2020,{ }^{5,6}$ The median survival of MPM ranges from 8 to 14 months from the diagnosis..$^{5-7,10}$ Male is predominant of $4: 1 .{ }^{10}$ Four main histological subtypes of MPM are classified as the following : 1 ) epithelioid ( most favourable prognosis with a median survival of 13.1 months), 2) sarcomatoid(worst outcomes with a median survival of 4 months), 3) biphasic or mixed, and 4) desmoplastic. ${ }^{5,6,10}$

\section{Pathogenesis}

Prolonged exposure to respirable asbestos fibers triggers an increase in inflammatory cytokines and reactive oxygen species (ROS) in the pleural microenvironment, both of which facilitate MPM carcinogenesis. ${ }^{11,12}$ Naturally, asbestos occurs in the form of silicate mineral with two different forms : 1) curly serpentine fibers of chrysotile or " white" asbestos and 2) sharp, needle-like fibers of amphibole asbestos. Amphibole asbestos is divided into :3) crocidolite (blue) asbestos, 4) amosite (brown) asbestos, 5) anthophyllite, 6) actinolite, and 7) tremolite. The risk of MPM development is associate with the type of fibers and heaviness and duration of exposure. ${ }^{5}$

Nevertheless, MPM is characterized by a low mutation load, ${ }^{13}$ with the most frequently mutated genes involved in MPM pathogenesis, " tumor suppressors (BAP1, CDKN2A, LATS2, NF2). ${ }^{14}$ After asbestos fibers are inhaled and migrate to the pleural 
space causing pleural irritation and a repeated cycle of tissue damage and repair. Asbestos fibers that penetrate mesothelial cells that cause cell mitosis interference, generating DNA mutation, and altering chromosome structure. These mesothelial cells release inflammatory cytokines, such as platelet-derived growth factor (PDGF), tumor growth factor- $\beta$ (TGF- $\beta$ ), and vascular endothelial growth factor (VEGF) that facilitate tumor growth. ${ }^{9}$ Asbestos fibers also induce the phosphorylation various protein kinases (extracellular signalregulated kinase 1 and 2 and mitogen-activated protein) that increases the expression of proto-oncogenes and facilitating abnormal cellular proliferation, ${ }^{15}$ In PMP tumor, there is reduced expression of key molecules in the p53 tumor-suppressor gene pathway (p14, p16, and NF2-MERLIN). ${ }^{15}$ There are deletions and loss mutations of BAP1 (BRCA-associated protein 1), CFAP45 (cilia and flagella associated protein 45), DDX3X, DDX51,RR2 (ryanodine receptor 2), SETDB1 (set domain bifurcated 1), SETD2 (set domain containing 2), and ULK2 (unc-like autophagy activating kinase) ${ }^{16}$ Nevertheless, MPM has a low frequency of protein-altering mutations, approximately 25 mutations per tumor, ${ }^{17}$ and contributing to the limitations of the potential for molecular targeted therapy. ${ }^{18}$

\section{Clinical manifestations, investigation and diagnosis}

Chest radiological imaging should be performed in all MPMsuspected patients for diagnostic and staging information. Characteristically radiological findings may be nodular pleural thickening, pleural plaques, a localized pleural-mass lesion, pleural effusion, irregular fissural thickening, or loss of hemithoracic volume. Nevertheless, further radiologically imaging tools, such as bedside chest ultrasonography, computed tomographic imagingare usually required due to insensitive and nonspecific chest radiographic features in general. ${ }^{19-22}$ Positron-emission technology-computed tomography (PET-CT) combines high-resolution computed tomographic (CT) scanning injected with a radioactive metabolic tracer (such as 18-fluoro-deoxy-glucose (FDG)) or magnetic resonance imaging (MRI). ${ }^{23-25}$ Nevertheless, PET-CT has low sensitivity for the diagnosis of extrapleural lesions due to its poor spatial resolution. ${ }^{26}$ In addition to CT scanning of the chest, surgical information by videoassisted thoracoscopic surgery (VATS) plus mediastinoscopy which is the current gold standard for staging in MPM and is superior to CT for assessing tumor size and suspected nodal metastasis, ${ }^{27,28}$ is the consensus by using the International Mesothelioma Interest Group staging classification, ${ }^{29}$ whereas the European Pneumological Society, ${ }^{30}$ recommends using the tumor-nodes-metastases (TNM) classification of the Union for International Cancer Control (UICC). ${ }^{3}$ VATS reveals the sensitivity and specificity of $95 \%-98 \%$ and $100 \%$, respectively and enables the removal of specimens under visual observation, as well as pleurodesis in the same procedure. ${ }^{28}$ VATS also enables the assessment of its respectability. ${ }^{32}$

By pleural puncture and cytological diagnosis, tumor cells are identified in pleural effusion more than $50 \%$ of patients with MPM, with the likelihood of positive cytology depending on the MPM subtypewith the limited cytological diagnosis. ${ }^{33}$ Percutaneous needle biopsy without image guidance reveals the sensitivity and specificity of $7 \%-47 \%$ and $100 \%$, respectively. ${ }^{28}$

There are several circulating tumor proteins identified in patients with MPM, such as mesothelin (MSLN, a cell-surface glycoprotein expressed by mesothelial cells), ${ }^{34-36}$ osteopontin(an integrin-binding protein implicated in cell-matrix interaction and overexpressed in several types of cancers), ${ }^{37,38}$ and fibulin-3 (a secreted glycoprotein implicated in cell proliferation and migration correlated with advanced disease, also identified in pleural fluid). ${ }^{39,} 40$ Soluble mesothelinrelated peptide (SMRP), a soluble form of mesothelin is secreted by the tumor cells into the blood circulation. ${ }^{41-43}$ SMRP seems to be effective in predicting response to chemotherapy and patient survival although it is not specific for MPM and cannot be considered an early diagnostic biomarkers for MPM surveillance program. ${ }^{41,44-48}$ Several studies revealed that plasma osteopontin is a more reliable and stable biomarker than serum osteopontin and the data involving its diagnostic accuracy are inadequate. ${ }^{49-51}$ Combined measurement of circulating SMRP and osteopontin is not more informative than measurement of circulating SMRP alone, ${ }^{49,50,52,53}$ Several previous studies demonstrated that fibulin-3 was not beneficial for differentiating patients with MPM from patients affected by other diseases, ${ }^{54}$ and was not effective as mesothelin..$^{55}$

Other biomarkers for detecting MPM are inflammatory and angiogenic factors (High Mobility Group B1 (HMGB1) and VEGF), biomarkers of oxidative stress (Reactive Oxygen Species (ROS) and Reactive Nitrogen Species (RNS)), circulating micro-ribonucleic acids (miRNAs), circulating tumor deoxyribonucleic acid (ctDNA), circulating methylated deoxyribonucleic acid (circulating methylated DNA), and circulating tumor cells (CTCs).A previous study indicated that HMGB1 and its receptors were highly expressed in MPM cell lines and tissues. ${ }^{56}$ VEGF, a key stimulator of tumor neoangiogenesis, is overexpressed in MPM tissues. ${ }^{57-59}$ In comparison to patients with lung cancer or non-malignant pleural diseases, circulating VEGF levels are increased in pleural effusions of patients with MPM. ${ }^{60}$ ROS and RNS are key mediators of asbestos toxicity. ${ }^{61}$ Bronchoalveolar lavage (BALF) of patients with asbestos exposure demonstrated an increase in various biomarkers of inflammation and altered ROS and iron homeostasis (i.e., iron, lactoferrin, ferritin, transferrin, and transferrin receptors)compared to controls. ${ }^{62} \mathrm{~A}$ previous study on the levels of miR-103a-3p and miR-30e-3p in extracellular vesicles demonstrated that the combination of these two biomarkers discriminated patients with MPM from asbestos-exposed controls with a sensitivity of $95.5 \%$ and specificity of $80 \%$ and were confirmed by normalizing the data to RNU48, miR-99a, miR-638, miR-720, and miR-1274a. ${ }^{63}$ In consideration, these miRNAs could be biomarkers of asbestos exposure rather than disease. Upregulation ofmiR-2053 could be a good prognostic biomarker of MPM. ${ }^{64}$ Detection ofctDNA variants in patients with MPM could be a potential biomarker for the diagnosis of MPM. ${ }^{65-67}$ Detection ofchanges in ctDNA methylation could be an early diagnostic and prognostic tool of MPM ${ }^{68} \mathrm{CTCs}$ countsin the blood circulation is very low at the early stage and increases in advanced stage of cancer. ${ }^{696}$ CTC-chip " test that developed by Chikaishi et al. demonstrated better performance than CELLSEARCH®test that is approved by the United States Food and Drug Administration (US FDA). ${ }^{70,71}$

Chest pain is common and usually heavy and dull and sometime called a "dragging" sensation, 5,72 Breathlessness is also common and is usually due to a pleural effusion that is identified in about $70 \%$ of MPM patients in early stage of disease. ${ }^{73}$ Other clinical features include anorexia, weight loss, malaise, sweats, and fatigue. .,6,72-78 $^{-1}$

\section{Management}

Systemic treatment options include targeted therapy, chemotherapy, and radiotherapy, provided separately or as part of multimodality treatment, whereas surgery is controversial and restricted to patients 
with good functional status and early stage disease. Treatment of MPM patients should be discussed at a specialist mesothelioma multidisciplinary team meeting..$^{5,79,80}$ There are several unanswered questions regarding chemotherapy in MPM patients, such as whether immediate chemotherapy is more effective than chemotherapy delayed until the appearance of clinical manifestations, the unclear optimum number of cycles of chemotherapy, the role of maintenance pemetrexed, and the benefit from continuing pemetrexed as maintenance therapy following an initial response. ${ }^{81}$ Nevertheless, chemotherapy is the only treatment modality that improve survival in MPM patients, but there is still no curative treatment for MPM.A recent study demonstrated that bevacizumab, an anti-VEGF monoclonal antibody in targeted therapy was effective in the treatment of MPM and is warranted alongside first-line standard chemotherapy in patients with unresectable MPM. ${ }^{82,83}$ Radiotherapy is beneficial in two main types : 1) as a palliative treatment, and 2) an adjuvant to chemotherapy and surgery in the context of trimodality treatment.In consideration the benefit of surgery, there is a need for large-scale randomized trial data to investigate its efficacy. ${ }^{5,84,85}$ For controlling pleural fluid, chemical pleurodesis is a procedure that can obliterate the pleural space and preventing pleural fluid re-accumulation. Good palliative care foe MPM patients is emphasized by the British Thoracic Society, the European Respiratory Society, and the International Mesothelioma Interest Group to manage clinical symptoms, emotional or spiritual, and psychological support for maintaining quality of life and dignified life. ${ }^{5,6}$

\section{Discussion}

Several circulating biomarkers are investigated for screening and detection of MPM, such as mesothelin, osteopontin, fibulin-3, HMGB1, VEGF, ROS, RNS, miRNAs, ctDNA. ${ }^{34-68}$ Additionally, " CTC-chip " test developed by Chikaishi et al revealed better results than "CELLSEARCH ${ }^{\circledR}$ " test that approved by the US FDA. CTCs counts increases in the MPM patients with advanced stage. ${ }^{69}$ The mentioned miRNAs are beneficial biomarkers of asbestos exposure rather than advanced stage MPM. Detection of changing ctDNA methylation could be beneficial in early diagnosis and prognosis of MPM, ${ }^{68}$ whereas upregulation of miR-2053 could be a good prognostic biomarker of MPM. ${ }^{64}$ For detection of asbestos toxicity, both ROS and RNS are the key mediators. ${ }^{61}$ Breathlessness, identified in around $70 \%$ of MPM patients and chest pain are common symptoms in addition to weight loss, anorexia, sweats, fatigue, and malaise. ${ }^{5,6,72-78}$.

Surgery is controversial and limited to MPM patients with early stage disease and good functional status. The efficacy of surgery is urgently needed for large-scale randomized trial data. ${ }^{5,84,85}$ Although there is no curative treatment, chemotherapy is the only treatment modality that improve MPM patients' survival. Nevertheless, there are still several questionable issues, e.g. whether immediate chemotherapy is more effective than chemotherapy delayed until the appearance of clinical features, the unclear suitable number of the cycles of chemotherapy, the role of maintenance pemetrexed, and the benefit from continuing pemetrexed as maintenance therapy following an initial response, ${ }^{81}$ whereas the roles of radiotherapy are only two main types, palliative treatment and adjuvant to chemotherapy and surgery in trimodalitytreatment. Numerous novel therapeutic agents for MPM have been studied, such as EGFR antagonist targeted therapy and PDGF inhibitor targeted therapy. ${ }^{86-89}$ Mesothelin-targeted treatments, such as chimeric antigen receptor T-cells targeted to mesothelin, mesothelin tumor vaccine (CRS-207), anti-mesothelin immunotoxins (e.g. SSIP), and mesothelin-specific monoclonal antibodies (e.g. amatuximab) are another interesting area in MPM treatment. ${ }^{90-94}$ Checkpoint inhibitors, the immunotherapeutic agents, such as pembrolizumab and tremelimumab have demonstrated prolonged disease stability and impressive disease control rates when used as first-, second-, and third-line treatment, ${ }^{95-99}$ Further exploration in synergistic effects of the combination of chemotherapy with immunotherapy or multiple immunotherapy agents in MPM treatment. ${ }^{100-102}$ In fact, the latency of MPM can be as long as 60 years, the patient's occupational history must be investigated extensively by an properly qualified health personnel and it must be kept in mind that MPM can be caused by short-term, low-level asbestos exposure. ${ }^{103,104}$

\section{Conclusion}

MPM, a complex disease can cause important morbidity and mortality. MPM remainsa diagnostic and therapeutic challenge ambulatory and in-hospital care. There is potential for the development of biomarkers and radiological imaging in the years to come. Several novel therapeutic options are under investigated and it is likely to involve highly personalized treatment. Its incidence has been constant in recent years and expect to decrease in the next decade.

\section{Acknowledgments}

Dr. Attapon Cheepsattayakorn conducted the study framework and wrote the manuscript. Associate Professor Dr. Ruangrong Cheepsattayakorn contributed to scientific content and assistance in manuscript writing. Both authors read and approved the final version of the manuscript.Both Assistant Professor Dr. SupawanManosoontorn and Dr. Vijaya Bhakskara Reddy Mutha were responsible for the reference citation search.

\section{Conflicts of interest}

The authors declare there are no conflicts of interest.

\section{Funding}

None.

\section{References}

1. Delgermaa V, Takahashi K, Park E, et al. Global mesothelioma deaths reported to the World Health Organization between 1994 and 2008. World Health Organization. 2011;89:716-724C.

2. Woitowitz H, Hillerdal G, Galvarezos A, et al. Risko undEinflussfaktoren des diffusenmalignen Mesotheliomas (DMM) Forschungsberichtsrehe FB 698 BundesanstaltfürArbeitsschutz. 1993.

3. Robinson BM. Malignant pleural mesothelioma:an epidemiological perspective. Ann Cardiothorac Surg. 2012;1(4):491-496.

4. Vogelzang NJ, Rusthven JJ, Symanowski J, et al. Phase III study of pemetrexed in combination with cisplatin versus cisplatin alone in patients with malignant pleural mesothelioma. J Clin Oncol. 2003;21(4):26362644.

5. British Thoracic Society Standards of Care Committee. BTS statement on malignant mesothelioma in the UK, 2007. Thorax. 2007; 62:Suppl 2, ii1-ii19.

6. Scherpereel A, Astoul P, Baas P, et al. Guidelines of the European Respiratory Society and the European Society of Thoracic Surgeons for the management of malignant pleural mesothelioma. Eur Respir J.2010;35(3):479-495. 
7. Yates D, Corrin B, Stidolph P, et al. Malignant mesothelioma in south east England:clinicopathological experience of 272 cases. Thorax. 1997;52(6):507-512.

8. Howel D, Arblaster L, Swinburne L, et al. Routes of asbestos exposure and the development of mesothelioma in an English region. Occup Environ Med.1997;54(6):403-409.

9. Sekido Y. Molecular pathogenesis of malignant mesothelioma. Carcinogenesis.2013;34(7):1413-1419.

10. Beckett P, Edwards J, Fennell D, et al. Demographics, management and survival of patients with malignant pleural mesothelioma in the National Lung Cancer Audit in England and Wales. Lung Cancer. 2015;88(3):344348.

11. Carbone M, Yang H. Molecular pathways:targeting mechanisms of asbestos and erionite carcinogenesis in mesothelioma. Clin Cancer Res. 2012;18(3):598-604.

12. Bograd AJ, Suzuki K, Vertes E, et al. Immune response and immunotherapeutic interventions in malignant pleural mesothelioma. Cancer ImmunolImmunother. 2011;60(11):1509-1527.

13. Chalmers ZR, Connelly CF, Fabrizio D, et al. Analysis of 100,000 human cancer genomes reveals the landscape of tumor mutational burden Genome Med. 2017;9(1):34.

14. Bott M, Brevet M, Taylor BS, et al. The nucleardeubiquitinase BAP1 is commonly inactivated by somatic mutations and 3p21.1 losses in malignant pleural mesothelioma. Nat Genet. 2011;43(7):668-672.

15. Robinson BW, Lake RA. Advances in malignant mesothelioma. $N$ Eng $J$ Med. 2005;353(15):1591-1603.

16. Illei PB, Rusch VW, Zakowski MF, et al. Homozygous deletion of CDKN2A and codeletion of the methylthioadenosine phosphorylase gene in the majority of pleural mesotheliomas. Clin Cancer Res.2003;9(6):2108-2113.

17. Guo G, Chmielecki J, Goparaju C, et al. Whole-exome sequencing reveals frequent genetic alterations in BAP1, NF2, CDKN2A, and CUL1 in malignant pleural mesothelioma. Cancer Res. 2015;75(2):264-269.

18. Torti D, Trusolino L. Oncogene addiction as a foundational rationale for targeted anti-cancer therapy:promises and perils. EMBO Mol Med. 2011;3(11):623-636.

19. Salonen O, Kivisaari L, Standertskjöld-Nordenstam C-G, et al. Computed tomography of pleural lesions with special reference to the mediastinal pleura. Acta Radiol Diagn (Stockh).1986;27(5):527-531.

20. Havelock T, Teoh R, Laws D, et al. Pleural procedures and thoracic ultrasound: British Thoracic Society pleural disease guidelines 2010 . Thorax. 2010; 65:Suppl 2:i61-i76.

21. Qureshi NR, Rahman NM, Gleeson FV. Thoracic ultrasound in the diagnosis of malignant pleural effusion. Thorax. 2009;64(2):139-143.

22. Hierholzer J, Luo L, Bittner RC, et al. MRI and CT in the differential diagnosis of pleural disease. Chest. 2000;118(3):604-609.

23. Benard F, Sterman D, Smith RJ, et al. Metabolic imaging of malignant pleural mesothelioma with fluorodeoxyglucose positron emission tomography. Chest. 1998;114:713-722.

24. YildirimH,Metintas M,EntokE, etal.Clinicalvalue offluorodeoxyglucosepositron emission tomography/computed tomography in differentiation of malignant mesothelioma from asbestos-related benign pleural disease:an observational pilot study. J Thorac Oncol. 2009;4(12):1480-1484.

25. Treglia G, Sadeghi R, Annunziata S, et al. Diagnostic accuracy of $18 \mathrm{~F}-$ FDG-PET and PET/CT in the differential diagnosis between malignant and benign pleural lesions:a systematic review and meta-analysis. Acad Radiol. 2014;21(1):11-20.
26. Steward D, Waller D, Edwards J, et al. Is there a role for pre-operative contrast-enhanced magnetic resonance imaging for radical surgery in malignant pleural mesothelioma ?. Eur JCardiothorac Surg. 2003;24(6); 1019-1024.

27. Heelan R, Rusch V, Begg C, et al. Staging of malignant pleural mesothelioma:comparison of $\mathrm{CT}$ and MR imaging. Am $J$ Roentgenol. 1999;172(4):1039-1047.

28. Attanous R, Gibbs A. The comparative accuracy of different pleural biopsy techniques in the diagnosis of malignant mesothelioma. Histopathol. 2006;53(3):340-344.

29. Rusch VW. A proposed new international TNM staging system for malignant pleural mesothelioma. Chest. 1995;108(4):1122-1128.

30. Scherpereel A, Astoul P, Baas P, et al. Guidelines of the European Respiratory Society and the European Society of Thoracic Surgeons for the management of malignant pleural mesothelioma. Eur Respir J. 2010;35(3):479-495.

31. Wittekind C, Meyer H. TNM Klassifikation maligner Tumoren. $6^{\text {th }}$ ed. Ch Wittekind, HJ Meyer, F Bootz, editors. Weinheim:Wiley-VCH; 2010.

32. Hasegawa S, Kondo N, Matsumoto S, et al. Practical approaches to diagnose and treatment to malignant pleural mesothelioma: a proposal for diagnostic total parietal pleurectomy. Int J Clin Oncol. 2012;17(1):33-39.

33. Husain A, Colby T, Ordonez N, et al. Guidelines for pathologic diagnosis of malignant mesothelioma. Arch Pathol Lab Med. 2013;137(5):647-67.

34. Pastan I, Hassan R. Discovery of mesothelin and exploiting it as a target for immunotherapy. Cancer Res. 2014;74(11):2907-2912.

35. Chang K, Pai LH, Batra JK, et al. Characterization of the antigen (CAK1) recognized by monoclonal antibody $\mathrm{K} 1$ present on ovarian cancers and normal mesothelium. Cancer Res. 1992;52(1):181-186.

36. Chang K, Pai LH, Pass H, et al. Monoclonal antibody K1 reacts with epithelial mesothelioma but not with lung adenocarcinoma. Am J Surg Pathol. 1992;16(3):259-68.

37. Coppola D, Szabo M, Boulware D, et al. Correlation ofosteopontin protein expression and pathological stage across a wide variety of tumor histologies. Clin Cancer Res. 2004;10(1):184-190.

38. Pass HI, Lott D, Lonardo F, et al. Asbestos exposure, pleural mesothelioma, and serum osteopontin levels. N Engl J Med. 2005;353 (15):1564-1573.

39. Creaney J, Dick IM, Robison BW. Comparison of mesothelin and fibulin-3 in pleural fluid and serum as markers in malignant mesothelioma. Curr Opin Pulm Med. 2015;21(4):352-356.

40. Pass HI, Levin SM, Harbut MR, et al. Fibulin-3 as a blood and effusion biomarker for pleural mesothelioma. N Engl J Med. 2013;368(2):190.

41. Scholler N, Fu N, Yang Y, et al. Soluble member(s) of the mesothelin/ megakaryocyte potentiating factor family are detectable in sera from patients with ovarian carcinoma. Proc NatlAcad Sci USA. 1999;96(20):11531-11536.

42. Hollevoet K, Reitsma JB, Creaney J, et al. Serum mesothelin for diagnosing malignant pleural mesothelioma: an individual patient data meta-analysis. J Clin Oncol. 2012;30(13):1541-1549.

43. Rai AJ, Flores RM, Mathew A, et al. Soluble mesothelin-related peptides (SMRP) and osteopontin as protein biomarkers for malignant mesothelioma: analytical validation of ELISA-based assays and characterization at mRNA and protein levels. Clin Chem Lab Med. 2010;48(2):271-278.

44. Beyer HL, Geschwindt RD, Glover CL, et al. MESOMARK:A potential test for malignant pleural mesothelioma. Clin Chem. 2007 53(4):666-672.

45. Ordonnez NG. Value of mesothelin immunostaining in the diagnosis of mesothelioma. Mod Pathol. 2003;16(3):192-197. 
46. Cristaudo A, Foddis R, Vivaldi A, et al. Clinical significance of serum mesothelin in patients with mesothelioma and lung cancer. Clin Cancer Res. 2007;13(17):5076-5081.

47. Grigoriu BD, Scherpereel A, Devos P, et al. Utility of osteopontin and serum mesothelin in malignant pleural mesothelioma diagnosis and prognosis assessment. Clin Cancer Res. 2007;13 (10):2928-2935.

48. Schneider J, Hoffmann H, Dienemann H, et al. Diagnostic and prognostic value of soluble mesothelin-related proteins in patients with malignan pleural mesothelioma in comparison with benign asbestosis and lung cancer. J Thorac Oncol. 2008;3(11):1317-1324.

49. Cristaudo A, Bonotti A, Simonini S, et al. Combined serum mesothelin and plasma osteopontin measurements in malignant pleural mesothelioma. $J$ Thorac Oncol. 2011;6(10):1587-1593.

50. Creaney J, Yeoman D, Musk AW, et al. Plasma versus serum levels of osteopontin and mesothelin in patients with malignant mesotheliomawhich is best ?. Lung Cancer. 2011;74(1):55-60.

51. FoddisR, Bonotti A, Landi S, et al. Biomarkers in the prevention and follow-up of workers exposed to asbestos. J Thorac Dis. 2018;10:S360S368

52. Hollevoet K, Nackaerts K, Gosselin R, et al. Soluble mesothelin, megakaryocyte potentiating factor, and osteopontin as markers of patient response and outcome in mesothelioma. J Thorac oncol. 2011;6(11):1930 1937.

53. Wheatley-Price P, Yang B, Patsios D, et al. Soluble mesothelinrelated peptide and osteopontin as markers of response in malignant mesothelioma. J Clin Oncol. 2010;28(20):3316-3322.

54. Kirschner MB, Pulford E, Hoda MA, et al. Fibulin-3 levels in malignant pleural mesothelioma are associated with prognosis but not diagnosis. $\mathrm{Br}$ J Cancer. 2015;113(6):963-969.

55. Creaney J, Dick IM, Meniawy TM, et al. Comparison of fibulin-3 and mesothelin as markers in malignant mesothelioma. Thorax. 2014;69(10):895-902.

56. Jube S, Rivera ZS, Bianchi ME, et al. Cancer cell secretion of the DAMP protein HMGB1 supports progression in malignant mesothelioma. Cancer Res. 2012;72(13):3290-3301

57. Kumar-Singh S, Weyler J, Martin MJ, et al. Angiogenic cytokines in mesothelioma:a study of VEGF, FGF-1 and-2, and TGF-beta expression. J Pathol. 1999;189:72-78.

58. Strizzi L, Catalano A, Vianale G, et al. Vascular endothelial growth factor is an autocrine growth factor in human malignant mesothelioma. J Pathol. 2001;193(4):468-475.

59. Demirag F, Unsal E, Yilmaz A, et al. Prognostic significance of vascular endothelial growth factor, tumor necrosis, and mitotic activity index in malignant pleural mesothelioma. Chest. 2005;128(5):3382-3387.

60. Hirayama N, Tabata C, Tabata R, at al. Pleural effusion VEGF levels as a prognostic factor of malignant pleural mesothelioma. Respir Med. 2011;105(1):137-142.

61. Shukla A, Gulumian M, Hei TK, et al. Multiple roles of oxidants in the pathogenesis of asbestos-induced diseases. Free Radic Biol Med. 2003;34(9):1117-1129.

62. Ghio AJ, Stonehuerner J, Richards J, et al. Iron homeostasis in the lung following asbestos exposure. Antioxid Redox Signal. 2008;10(2):371377.

63. Cavalleri T, Angelici L, Favero C, et al. Plasmatic extracellular vesicle microRNAs in malignant pleural mesothelioma and asbestos-exposed subjects suggest a 2-miRNA signature biomarkers of disease. PLOS ONE. 2017;12(5):e0176680.
64. Matboli M, Shafei AE, Ali MA, et al. Clinical significance of serum DRAM1 mRNA, ARSA mRNA, has-miR-2053 and lncRNA-RP186D1.3 axis expression in malignant pleural mesothelioma. $J$ Cell Biochem. 2019;120(3):3203-3211.

65. Fan HC, Blumenfeld YJ, Chikara U, et al. Analysis of the size distributions of fetal and maternal cell-free DNA by paired-end sequencing. Clin Chem. 2010;56(8):1279-1286.

66. Corcoran RB, Chabner BA. Application of cell-free DNA analysis to cancer treatment. $N$ Engl J Med. 2018;379(18):1754-1765.

67. Leon SA, Shapiro B, Sklaroff DM, et al. Free DNA in the serum of cancer patients and the effect of therapy. Cancer Res. 1997;37(3):646-650.

68. Kanherkar RR, Bhatia-Dey N, et al. Epigenics across the human lifespan. Front Cell Dev Biol. 2014;2:49.

69. Young R, Pailler E, Billiot F, et al. Circulating tumor cells in lung cancer Acta Cytol. 2012;56:655-660.

70. Su DW, Nieva J. Biophysical technologies for understanding circulating tumor cell biology and metastasis. Trans Lung Cancer Res. 2017;6(4):473485.

71. Chikaishi Y, Yoneda K, Ohnaga T, et al. EpCAM-independent capture of circulating tumor cells with a ' universal CTC-chip'. Oncol Rep. 2017;37(1):77-82.

72. Clayson H, Seymour J, Noble. The experience of mesothelioma in Northern England. UK: University of Sheffield; 2007.

73. Rudd RM. Malignant mesothelioma. Br Med Bull. 2010;93:105-123.

74. Clayson H, Seymour J, Noble B. Mesothelioma from the patient's perspective. Hematol Oncol Clin North Am. 2005;19(6):1175-1190.

75. Nowak AK, Stockler MR, Byrne MJ. Assessing quality of life during chemotherapy for pleural mesothelioma: feasibility, validity, and results of using the European Organization for Research and Treatment of Cancer Core Quality of Life Questionnaire and Lung Cancer Module. J Clin Oncol. 2004;22(15):3172-3180.

76. van Zandwijk N, Clarke C, Henderson D, et al. Guidelines for the diagnosis and treatment of malignant pleural mesothelioma. J Thorac Dis. 2013;5(6):E254-E307.

77. Muers MF, Stephen RJ, Fisher P, et al. Active symptom control with or without chemotherapy in the treatment of patients with malignant pleural mesothelioma (MS01): a multicenter randomized trial. Lancet. 2008;371:1685-1694

78. O' Brien ME, Watkins $\mathrm{D}$, Ryan $\mathrm{C}$, et al. A randomized trial in malignant mesothelioma (M) of early (E) versus delayed (D) chemotherapy in symptomatically stable patients: the MED trial. Ann Oncol. 2006;17(2):270-275.

79. Mesothelioma Service Framework. Department of Health Lung Cancer and Mesothelioma Advisory Group. London: Department of Health; 2007.

80. Bibby AC, Williams $\mathrm{K}$, Smith $\mathrm{S}$, et al. What is a role of a specialist regional mesothelioma multidisciplinary team meeting ?. A service evaluation of one tertiary referral centre in the UK. BMJ Open. 2016;6(9):e012092.

81. van den Bogaert DP, Pouw EM, van Wijhe G, et al. Pemetrexed maintenance therapy in patients with malignant pleural mesothelioma. $J$ Thorac Oncol. 2006;1(1):25-30.

82. Zalcman G, Mazieres J, Margery J, et al. Bevacizumab for newly diagnosed pleural mesothelioma in the Mesothelioma Avastin Cisplatin Pemetrexed Study (MAPS):a randomized, controlled, open-label, phase 3 trial. Lancet. 2016;387:1405-1414.

83. Zauderer MG. A new standard for malignant pleural mesothelioma. Lancet. 2016;387:1352-1354. 
84. Treasure T, Sedrakyan A. Pleural mesothelioma:little evidence, still time to do trials. Lancet. 2004;364:1183-1185.

85. Clive AO, Taylor H, Dobson L, et al. Prophylactic radiotherapy for the prevention of procedure-tract metastases after surgical an large-bore pleural procedures in malignant pleural mesothelioma (SMART): a multicenter, open-label, phase 3, randomized controlled trial. Lancet Oncol. 2016;17:1094-1104.

86. Garland LL, Rankin C, Gandara DR, et al. Phase II study of erlotinib in patients with malignant pleural mesothelioma:a Southwest Oncology Group Study. J Clin Oncol. 2007;25(17):2406-2413.

87. Govindan R, Kratzke RA, Herndon JE, et al. Gefitinib in patients with malignant mesothelioma:a phase II study by the Cancer and Leukemia Group B. Clin Cancer Res. 2005;11(6):2300-2304.

88. Mathy A, Baas P, Dalesio O, et al. Limited efficacy of imatinib mesylate in malignant mesothelioma:a phase II trial. Lung Cancer. 2005;50(1):83-86.

89. Nowak AK, Millward MJ, Creaney J, et al. A phase II study of intermittent sunitinib malate as second-line therapy in progressive malignant pleural mesothelioma. J Thorac Oncol. 2012;7(9):1449-1456.

90. Hassan R, Schweizer C, Lu KF, et al. Inhibition of mesothelin-CA125 interaction in patients with mesothelioma by the anti-mesothelin monoclonal antibody MORAb-009:Implications for cancer therapy. Lung Cancer. 2010;68(3):455-459.

91. Hassan R, Kindler HL, Jahan T, et al. Phase II clinical trial of amatuximab, a chimeric antimesothelin antibody with pemetrexed and cisplatin in advanced unresectable pleural mesothelioma. Clin Cancer Res. 2014;20(23):5927-5937.

92. Hassan R, Sharon E, Thomas A, et al. Phase I study of the antimesothelin immunotoxin SSIP in combination with pemetrexed and cisplatin for front-line therapy of pleural mesothelioma and correlation of tumor response with serum mesothelin, megakaryocyte potentiating factor, and cancer antigen 125. Cancer. 2014;120(21):3311-3319.

93. Hassan R, Antonia SJ, Alley EW, et al. Mesothelin-targeted immunotherapy CRS-207 in combination with standard of care chemotherapy as treatment for malignant pleural mesothelioma (MPM). $J$ Clin Oncol. 2015;33(15):7565.
94. Beatty GL, Haas AB, Maus MV, et al. Mesothelin-specific chimeric antigen receptor MRNA-engineered T cells induce antitumor activity in solid malignancies. Cancer Immunol Res. 2014;2(2):112-120.

95. Calabro L, Morra A, Fonsatti E, et al. Tremelimumab for patients with chemotherapy-resistant advanced malignant mesothelioma:an openlabel, single-arm, phase II trial. Lancet Oncol. 2013;14(11):1104-1111.

96. Calabro L, Morra A, Fonsatti E, et al. Efficacy and safety of an intensified schedule of tremelimumab for chemotherapy-resistant malignant mesothelioma:an open-label, single-arm, phase II study. Lancet Respir Med. 2015;3(4): 301-309.

97. Alley EW, Molife LR, Santoro A, et al. Clinical safety and efficacy of pembrolizumab (MK-3475) in patients with malignant pleural mesothelioma:preliminary results from Keynote-028. Lancet Oncol. 2017;18(5):623-630

98. Kindler HL, Karrison T, Khattri A, et al. Phase II study of the anti-PD-1 antibody pembrolizumab in patients with malignant mesothelioma. $J$ Thorac Oncol. 2015;10:S630.

99. Cornelissen R, Scherpereel A, Peitro AD, et al. Randomized, doubleblind, placebo-controlled study of tremelimumab for second- and thirdline treatment for unresectable pleural or peritoneal mesothelioma. Annals of Oncology. 2014;25:iv370.

100. Emens LA, Middleton G. The interplay of immunotherapy and chemotherapy:harnessing potential synergies. Cancer Immunol Res. 2015;3(5):436-443

101. Calabro L, Morra A, Fonsatti E, et al. 16TiPA phase II study combining tremelimumab and durvalumab (MED14736) immunomodulating antibodies in mesothelioma patients:the NIBIT- MESO-1 study. Ann Oncol. 2015;26:Suppl 8:viii6-vviii.

102. Larkin J, Chiarion-Sileni V, Gonzalez R, et al. Combined nivolumab and ipilimumab or monotherapy in untreated melanoma. $N$ Engl J Med. 2015;373(1):23-34

103. Hillerdal G. Mesothelioma:cases associated with non- occupational and low-dose exposure. Occup Environ Med. 1999; 56(8):505-513.

104. Müller K, Dernbach A, Neumann V. Mesothelioma in academics. The Pathologist. 2003;24:109-113. 